\title{
X-RAY RAMAN SCATTERING IN H-BN OBSERVED BY SOFT X-RAY FLUORESCENCE SPECTROSCOPY*
}

\author{
J.J. Jia, T.A. Callcott \\ University of Tennessee \\ Knoxville, Tennessee 37996 \\ J.A. Carlisle, L.J. Terminello, A. Asfaw \\ Lawrence Livermore National Laboratory \\ Livermore, CA 94551 \\ D.L. Ederer \\ Tulane University \\ New Orleans, LA 70118 \\ F.J. Himpsel \\ IBM T.J. Watson Research Center \\ Yorktown Heights, NY 10598 \\ R.C.C. Perera \\ Advanced Light Source \\ Lawrence Berkeley Laboratory \\ University of California \\ Berkeley, CA 94720
}

March 1995

*This work was supported by the Director, Office of Energy Research, Office of Basic Energy Sciences, Materials Sciences Division, of the U.S. Department of Energy, under Contract No. DE-AC03-76SF00098. 


\section{DISCLAIMER}

This report was prepared as an account of work sponsored by an agency of the United States Government. Neither the United States Government nor any agency thereof, nor any of their employees, make any warranty, express or implied, or assumes any legal liability or responsibility for the accuracy, completeness, or usefulness of any information, apparatus, product, or process disclosed, or represents that its use would not infringe privately owned rights. Reference herein to any specific commercial product, process, or service by trade name, trademark, manufacturer, or otherwise does not necessarily constitute or imply its endorsement, recommendation, or favoring by the United States Government or any agency thereof. The views and opinions of authors expressed herein do not necessarily state or reflect those of the United States Government or any agency thereof. 


\section{DISCLAIMER}

Portions of this document may be illegible in electronic image products. Images are produced from the best available original document. 


\title{
$\mathrm{X}$-ray Raman scattering in $\mathrm{h}$-BN observed
}

by soft $X$-ray fluorescence spectroscopy

\section{J.J. Jia, T.A. Callcott}

University of Tennessee, Knoxville, TN 37996

J.A. Carlisle, L.J. Terminello, A. Asfaw

Lawrence Livermore National Laboratory, Livermore, CA 94551

\author{
D.L. Ederer \\ Tulane University, New Orleans, LA 70118 \\ F.J. Himpsel \\ IBM T.J. Watson Research Center, Yorktown Heights, NY 10598 \\ R.C.C. Perera \\ Lawrence Berkeley Laboratory, Berkeley, CA 94720
}

\begin{abstract}
Raman scattering of soft $\mathrm{x}$-rays is observed in $\mathrm{h}$-BN using monochromatic soft $\mathrm{x}$-rays just below the $\mathrm{B} \mathrm{K}$ absorption edge. The inelastic features are visible below threshold, track with the excitation energy, go through a resonance as the excitation is tuned to the B 1s core exciton energy, and finally evolve into normal fluorescence as the excitation is raised above the energy needed to excite the B 1s electron into the conduction band. The inelastic energy loss is identified as an excitation of valence $\sigma$ electrons into the $\pi^{*}$ valence exciton state; at resonance and above, $\pi-\pi^{*}$ transitions are also observed. At resonance, a sideband on the elastic peak is observed, which gives evidence of additional electronic and phonon loss processes. Very similar results have also been observed for $\mathrm{B}_{2} \mathrm{O}_{3}$.
\end{abstract}

PACS numbers : 78.70.En 
Interest in inelastic $\mathrm{x}$-ray scattering has been stimulated by the development of tunable synchrotron radiation sources. Observations to date have been largely confined to scattering of higher energy $x$-rays $(\sim \mathrm{keV}$ ) by bound electrons ( $\mathrm{L}$ shell electrons), $[1,2,3]$ though reports of an inelastic scattering process that conserves crystal momentum has been reported for $\mathrm{Si}$ diamond and graphite. [4,5,6] A general theory of inelastic processes for $\mathrm{x}$-rays has been developed by Åberg and Tulkki, which quite nicely accounts for inelastic scattering observed in localized atomic and molecular systems in the vicinity of the $\mathrm{K}$ absorption edge. $[7,8]$ Threshold phenomena observed in solids to date can be divided into two general classes depending on whether they involve states localized in crystal momentum (k-space) or real space. Recent attention has focused on the $k$-conserving processes in covalently bonded materials. In this paper, we describe inelastic scattering processes that occur well below threshold in hexagonal boron nitride (h-BN), a system with a strongly localized excited state. Similar phenomena have also been observed in $\mathrm{B}_{2} \mathrm{O}_{3}$ and other oxide systems and will be reported elsewhere.[9]

Using $x$-ray fluorescence detection and a large band-pass $(\approx 2 \mathrm{eV}$ ) monochromator, O'Brien et al.[10] measured large energy shifts in the fluorescence spectra of $h-B N$ and $\mathrm{B}_{2} \mathrm{O}_{3}$ for excitation of $\mathrm{B}$ 1s electrons into the core exciton states. The energy shift was discussed in terms of "spectator" versus "normal" emission. The excitonic electron, bound in a localized orbital near the core hole, acts as a spectator electron which changes the screening and causes an energy shift of the "normal" fluorescence spectrum. The contribution of phonon relaxation effects to the observed energy shifts were also discussed, but could not be separated unambiguously from the exciton effects. Substantial changes in the shape of the h-BN spectrum were also noted but not discussed in any detail. In other reports, the very large magnitude of the exciton resonance fluorescence peak in h-BN has been documented, but little attention was paid to the valence fluorescence spectrum.[11]

Equipped with a monochromator of much higher resolution and flux, we have been able to extend the near threshold studies in $h-B N$ and $\mathrm{B}_{2} \mathrm{O}_{3}$. Using monochromatized light of $0.3 \mathrm{eV}$ bandwidth near the $\mathrm{B}$ 1s threshold, we have discovered inelastic scattering at excitation energies many $\mathrm{eV}$ below the exciton resonance. For both materials, but more dramatically in $\mathrm{h}-\mathrm{BN}$, it generates a band of inelastically scattered photons which maintains a constant shape and moves in energy with the excitation energy. The intensity of the inelastic band increases to a maximum at the elastic excitonresonance-fluorescence peak, where additional structure is also observed. The inelastic phenomena are very much like previous observations in $\mathrm{Mn}$ and $\mathrm{Cu}$ at the $\mathrm{K}$ absorption edge.[2,3] These results provide a clear, unambiguous example of Raman scattering in the soft $\mathrm{x}$-ray range in very simple solid systems, and provide a textbook study of the inelastic scattering processes predicted by Åberg and Tulkki, as they evolve from normal inelastic Raman scattering below threshold, through resonant Raman scattering at the exciton or Ryderberg states to ordinary fluorescence at excitations well above threshold. In so doing, they also demonstrate that these phenomena should be present in solid systems, when weak screening permits the creation of localized states in the excitation spectrum.

$\mathrm{h}-\mathrm{BN}$ is a layered structure similar to graphite, in which strong $\mathrm{sp}^{2} \mathrm{\sigma}$-bonds are formed within the plane and $\pi$ orbitals extend out of the plane. The most useful calculations of its electronic properties are those of H. Ma et al. [12], who calculated separate projected densities of states for $\sigma$ and $\pi$ electrons, as well as exciton binding energies and other features of the absorption spectrum. It provides a well characterized system to study the inelastic scattering mechanism. 
Soft x-ray emission spectroscopy using photon excitation, i.e. soft x-ray fluorescence (SXF) has a number of unique properties. It provides information on the density of states that is selective for both chemical species and angular momentum and thus is especially valuable for the study of the electronic properties of complex materials. Both exciting and emitted photons penetrate many atomic layers, so that it is a bulk probe that is relatively insensitive to surface structure and contamination. Compared with electron excitation, photon excitation improves chemical selectivity, reduces damage of fragile materials, and eliminates the Bremstrahlung background which can badly obscure very weak spectra. Of greatest significance for this study, the photon in - photon out technique permits the study of the coupling of the excitation and emission processes that occurs near threshold in many materials. Traditionally, SXF spectroscopy has been severely limited by weak excitation sources and low radiative yields of the emission process. However, with the development of intense undulator-based synchrotron radiation excitation sources and improved spectrometers, many new experiments are now feasible.

The experiment was performed on beamline 8.0 at the Advanced Light Source (ALS). The beamline consists of a 89 period, $5 \mathrm{~cm}$ undulator, a spherical grating monochromator, and our soft $\mathrm{x}$ ray spectroscopy endstation. [13] The energy resolution of the monochromatic light used for excitation was set at $0.3 \mathrm{eV}$ for these experiments. Emitted radiation was measured with a Rowland circle type spectrometer utilizing large spherical gratings and a photon counting area detector. For these measurements, the spectrometer was operated at a resolution of $0.6 \mathrm{eV}$ for the boron $\mathrm{K}$ edge. The photon flux incident on the sample is monitored by photo-current from a gold coated screen inserted in the beam immediately upstream of the sample.

In Fig. 1, the SXF spectra of $h-B N$ attributable to inelastic-scattering and valence band fluorescence excited with photon energies near the boron $\mathrm{K}$ edge are shown. All spectra shown are normalized to the exciting photon flux, and labeled by the excitation energy used for each spectrum. (For clarity, the intensity of elastic peaks are reduced by a factor of 100 in the figure). The principal feature of the elastic peak is the very strong resonance observed at $192.0 \mathrm{eV}$ when boron $1 \mathrm{~s}$ electrons are excited into the exciton state derived from the boron $\pi^{*}$ orbitals. The intensity of the elastic peak increases by a factor of about 400 between the $186.8 \mathrm{eV}$ excitation and the resonance excitation at $192.0 \mathrm{eV}$.

For all excitation energies below the resonance energy of $192.0 \mathrm{eV}$, the inelastic spectrum shows two peaks ( $A$ and $B$ ) separated by $5 \mathrm{eV}$ and a much more intense elastic peak (E) at higher energy. These inelastic spectra exactly track the excitation energy, so that the upper peak of the inelastic spectrum (B) maintains a constant separation of $11 \mathrm{eV}$ below the elastic peak (E). At the resonance energy, a new feature appears in the inelastic spectrum in the form of a high energy shoulder (C) at about $2.5 \mathrm{eV}$ above peak B. At higher excitation energies, shoulder $\mathrm{C}$ disappears as the excitation moves off resonance, and then reappears as part of the normal fluorescence spectrum that is observed for excitation energies above $193.7 \mathrm{eV}$. For $193.7 \mathrm{eV}$ and higher excitation energies, all three spectral features $(A, B, C)$ are broadened and fixed in energy position. These spectra are characteristic of spectra that are generated when core electrons are excited into delocalized conduction band states, and the excitation and emission processes are effectively decoupled.

It is clear from Fig. 1 that both the elastic and inelastic peaks have a resonance maximum at $192 \mathrm{eV}$. In Fig. 2, the ratio of the peak B to the elastic peak (E) is shown as a function of excitation 
energy. In the inelastic scattering region below about $193 \mathrm{eV}$, the inelastic to elastic intensity ratio remains approximately constant except directly on the resonance maximum, where the elastic peak is much enhanced. When the excitation is at or above $193 \mathrm{eV}$, in the normal fluorescence region, the intensity of the fluorescence spectrum is decoupled from that of the elastic peak. The evolution of spectral features from inelastic scattering features that track the excitation energy to fixed-energy fluorescence features is clearly seen from these spectra.

In Fig. 3 , the spectrum of $h-B N$ is shown at resonance to emphasize the sideband that appear on the lower edge of the elastic peak. This sideband, indicated by arrows on the figure, gives evidence of electronic and/or phonon excitations as described further below. The sideband tails off for more than $7 \mathrm{eV}$ to the edge of the SXF spectrum.

Since SXF transitions obey dipole selection rules, the strong resonance transitions observed at the threshold for absorption are derived from boron p states that contribute to the conduction band at the conduction band minimum. In $h-B N$, the core exciton is derived from states at the conduction band edge, which are $\pi^{*}$ anti-bonding states derived from the boron $p_{z}$ orbitals.[12]

The inelastic spectra observed below the threshold resonance at $192 \mathrm{eV}$ in $\mathrm{h}-\mathrm{BN}$ can be understood to be of the type described by Åberg and Tulkki and commonly referred to as electronic Xray Raman spectra. To establish this interpretation, we need to understand the nature of the electronic - transitions responsible for the energy loss. The energy separation between the inelastic features and the elastic peak indicates that these transitions occur from valence band states to $\pi^{*}$ valence exciton states, consisting of an electron bound to a valence hole. The model we propose is illustrated in Fig. 4. Process (a) is the inelastic Raman scattering process below threshold; process (b) shows the resonant "spectator" fluorescence process that produces the same final state as the X-ray Raman process for excitation into the exciton resonance; and process (c) illustrates the normal fluorescence spectrum.

Fig. 5 compares h-BN SXF spectra below, at, and above resonance with the calculated boron p-like projected density of states for $\sigma$ and $\pi$ electrons. Spectral energies are aligned to the central maximum of the spectra and the calculated density of states. The comparison indicates that for Raman scattering below resonance, the measured spectra are due to transitions from o valence states to the $\pi^{*}$ valence exciton state, assumed to be approximately degenerate with the core exciton. The $\pi$ to $\pi^{*}$ transitions are forbidden in the inelastic scattering process, as would be expected on symmetry considerations for a two dimensional solid.[6] On resonance, a significant $\pi$ component is also seen in the spectrum. Two factors may contribute to this result. On resonance, the lifetime of the core hole is greatly increased, and the spectator fluorescence de-excitation channel is opened. Other work [10,15] has indicated that core-hole lifetimes in boron compounds are sufficiently long to permit phonon effects (lattice relaxation) to play a role in relaxing selection rules. For excitation between the exciton resonance and the conduction band minimum, the $\pi-\pi^{*}$ contributions are again suppressed. Finally, for excitation to the conduction band, both $\sigma$ and $\pi$ electrons contribute to the spectra.

Another feature of the resonance spectrum of $h-B N$ is of significant interest. A broad shoulder $\left(E^{\prime}\right)$ is observed extending to lower energy from the elastic peak. Because of the large energy losses involved, we suggest that this shoulder is associated with a decay process in which the excitonic electron decays into the conduction band, rather than into a valence exciton, though lattice relaxation effects may play a role.(see Fig. $4 b$ ). This shoulder is rather analogous to the low energy tailing 
routinely observed in SX emission spectra of metals and semiconductors, which is associated with electronic excitations which reduce the energy of the emitted photons.[14]

This research was supported by NSF grants DMR-9017996 and DMR-9017997, by the Science Alliance Tennessee Center of Excellence at the University of Tennessee, by the USDOE through its support of the Advanced Light Source at Lawrence Berkeley Laboratory under contract No. DE-AC03-76SF00098, and by the author's home institutions. One of us (A. Asfaw) would like to acknowledge $\mathrm{K}$ J. Redd and the Lawrence Livermore National Laboratory research collaboration program for historically black colleges and universities. 


\section{References}

1. C. J. Sparks, Phys. Rev. Lett. 33, 262 (1974).

2. P. Eisenberger, P. M. Platzman, and H. Winick, Phys. Rev. Lett. 36, 623 (1976).

3. J. P. Briand, D. Girard, V. O. Kostroun, P. Chevalier, K. Wohrer, and J. P. Mosse, Phys. Rev. Lett. 46, 1625 (1981).

4. J.E. Rubensson, D.R. Mueller, R. Shuker, D.L. Ederer, C.H. Zhang, J. Jia, and T.A. Callcott, Phys. Rev. Lett. 64, 1047 (1990); KE. Miyano, D.L. Ederer, T.A. Callcott, W.L. O'Brien, J.J. Jia, L. Zhou, Q.-Y. Dong, Y. Ma, J.C. Woicik and D.R. Mueller, Phys. Rev. B48, 1918 (1993).

5. Y. Ma, N. Wassdahl, P. Skytt, J. Guo, J. Nordgren, P.D. Johnson, J-E. Rubensson, T. Boske, W. Eberhardt, and S.D. Kevan, Phys. Rev. Lett. 69, 2598 (1993).

6. J.A. Carlisle, E. L. Shirley, E.A. Hudson, LJ. Terminello, T.A. Callcott, J.J. Jia, D.L. Ederer, R.C.C. Perera and FJ. Himpsel, Phys. Rev. Lett. 73, (Phys. Rev. Lett. in press).

7. T. Åberg and J. Tulkki, in Atomic Inner-shell Physics, ed. by B. Crasemann, Chap. 10, pp 419463 (Plenum, 1985) and references therein.

8. J. Tulkki, and T. Åberg, J. Phys. B15, L435 (1982); J. Tulkki, Phys. Rev. A27, 3375 (1983).

9. D.L Ederer, U. Diebold, T.A. Callcott, J.J. Jia, LJ. Terminello, J.A. Carlisle, and R.C.C. Perera, (paper on Ti oxides, to be submitted to Phys. Rev. B).

10. W.L. O'Brien, J.J. Jia, Q.-Y. Dong, T.A. Callcott, K.E. Miyano, D.L. Ederer, D.R. Mueller, C.-C. Kao, Phys. Rev. Lett. 70, 238 (1993).

11. J.A. Carlisle, R.C.C. Perera, J.H. Underwood, LJ. Terminello, E.A. Hudson, T.A. Callcott, J.J. Jia, and F.J. Himpsel (paper on buried monoloayers of BN, submitted to App. Phys. Lett.)

12. H. Ma, S.H. Lin, R.W. Carpenter, P. Rice and O.F. Sankey, J.Appl. Phys.73,7422 (1993).

13. J. J. Jia, T. A. Callcott, J. Yurkas, A. W. Ellis, F. J. Himpsel, M. G. Samant, J. Stöhr, D. L. Ederer, J. A. Carlisle, E. A. Hudson, L. J. Terminello, D. K. Shuh, and R. C. C. Perera. (To be published in Rev. Sci. Instrum.)

14. See, for example, P. Livins and S.E. Schnatterly, Phys. Rev. B37, 6731 (1988).

15. A. Mansour and S.E. Schnatterly, Phys. Rev. Lett. 59, 567 (1987).

16. G.D. Mahan, Phys. Rev. B15, 4587 (1977).

17. C-O. Almbladh, Phys. Rev. B16, 4343 (1977). 


\section{Figure Captions}

Fig. 1 The boron $\mathrm{K}$ soft $\mathrm{x}$-ray fluorescence spectra of $\mathrm{h}$-BN for excitation near threshold . Labelled features are discussed in the text. The elastic peak heights of all spectra are reduced by a factor of 100.

Fig. 2 h-BN spectral parameters vs. excitation energy. Square: the elastic peak (E) maximum (reduced by a factor of 100); Circle: the ratio of inelastic/fluorescence intensity maximum (peak B) to elastic peak maximum (peak E);

Fig. 3 Soft $x$-ray fluorescence spectra of $h-B N$ with excitation at the exciton resonance showing sidebands $(E)$ on elastic peak.

Fig. 4 Illustration of proposed model of electronic excitation processes occurring near threshold. (a) Inelastic scattering with below threshold excitation. (b) Spectator fluorescence with resonance excitation. (c) Fluorescence emission with excitation to delocalized states above conduction band minimum.

Fig. 5 Comparison of h-BN fluorescence spectra (190.0 eV, $192.0 \mathrm{eV}$ and $194.8 \mathrm{eV}$ excitations) with calculated $\sigma$ and $\pi$ densities of states (ref. 12). The $190 \mathrm{eV}$ and $192 \mathrm{eV}$ excited spectra have been shifted to higher photon energy by $2.2 \mathrm{eV}$ and $0.8 \mathrm{eV}$ respectively. Results indicate that only $\sigma$ electrons contribute to the inelastic scattering spectra observed with below threshold excitation. 


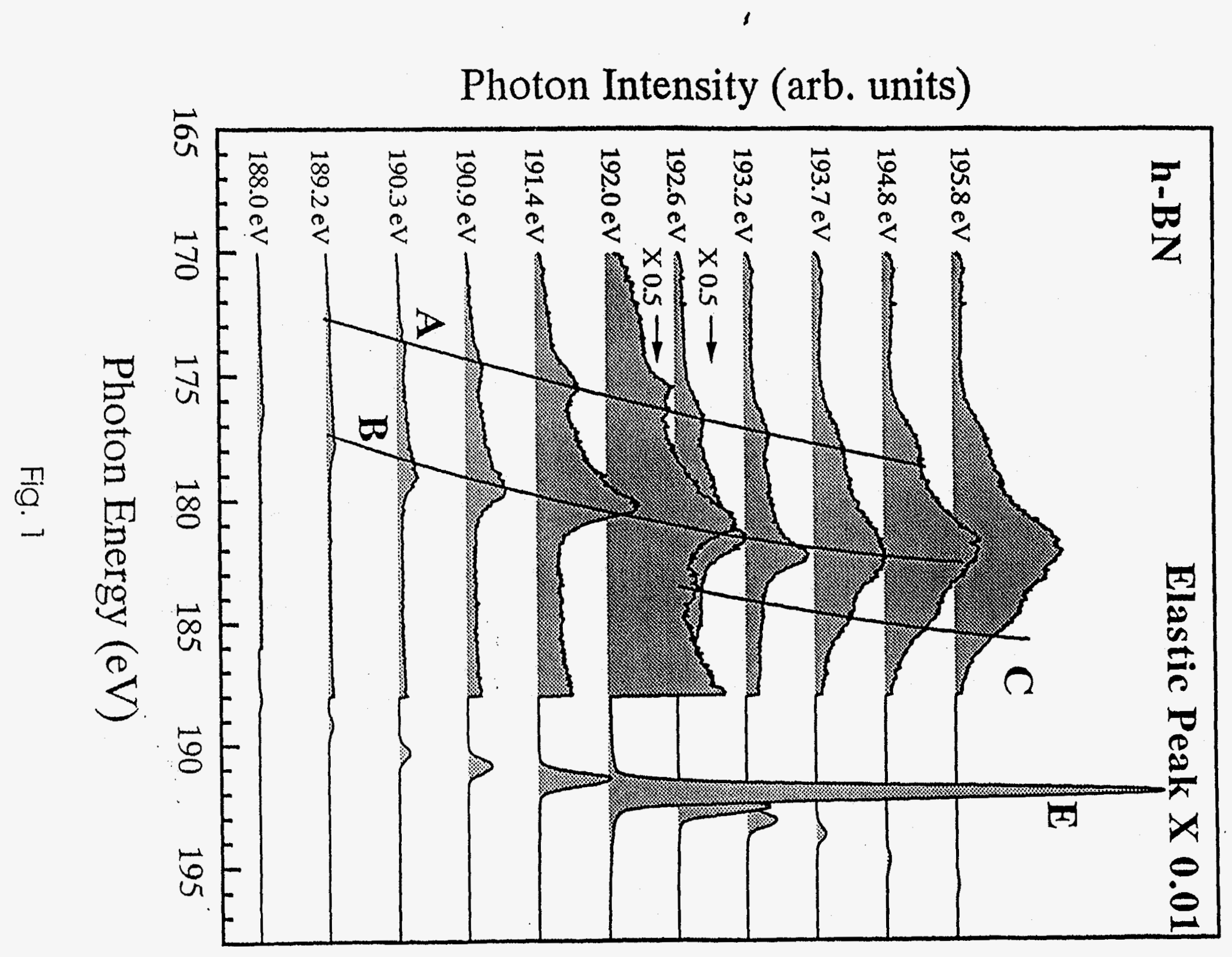




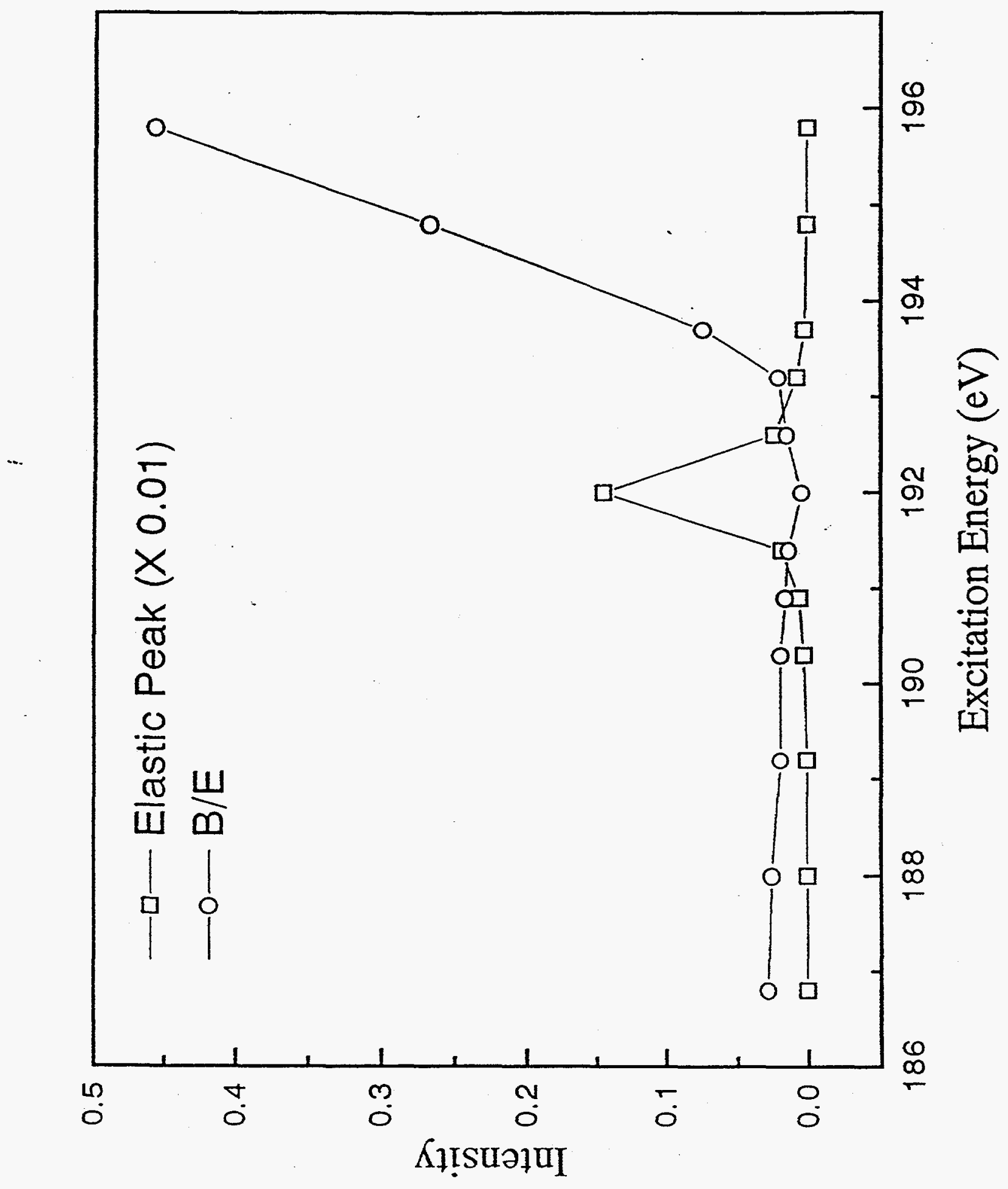

$\frac{N}{i}$ 


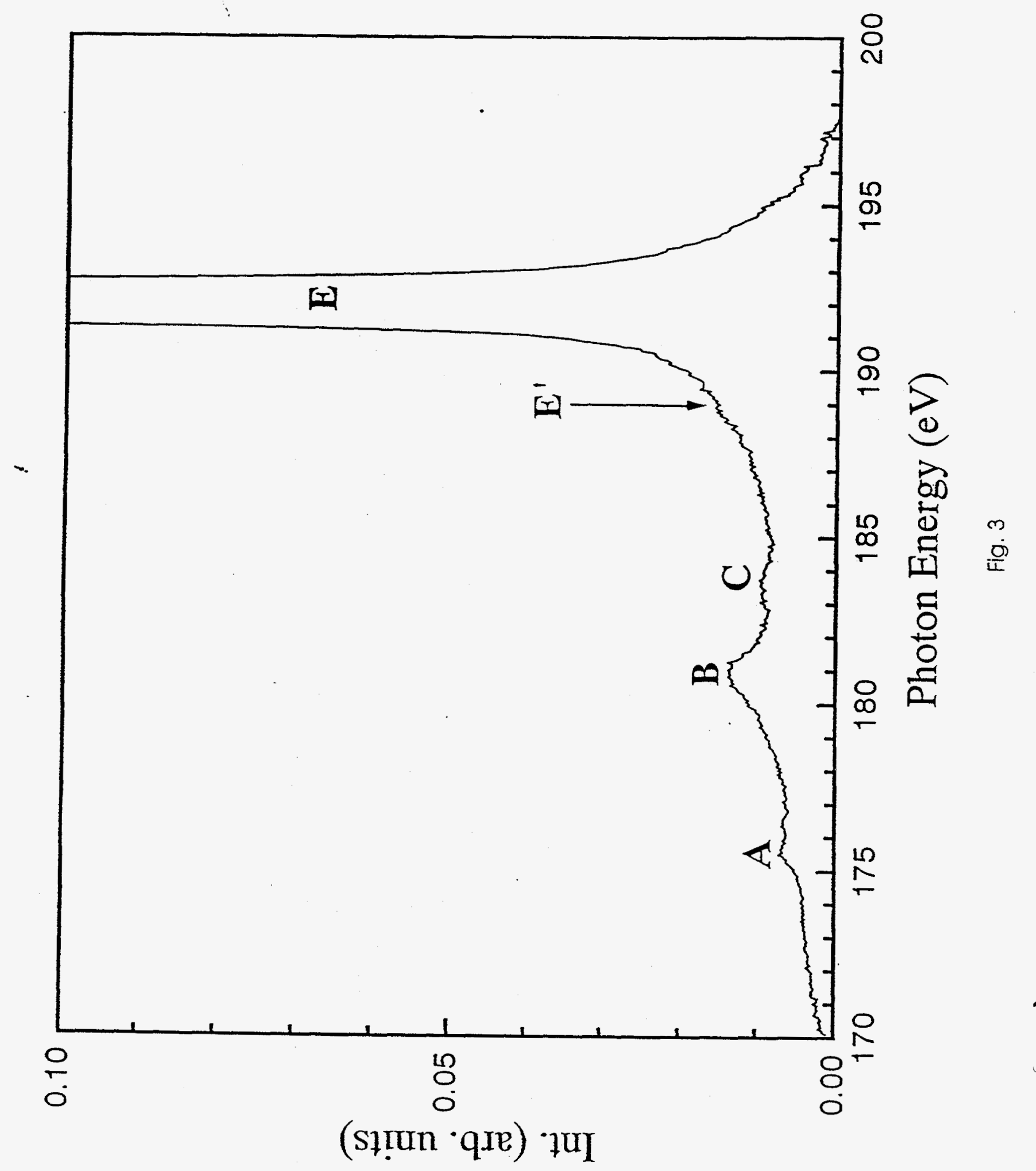




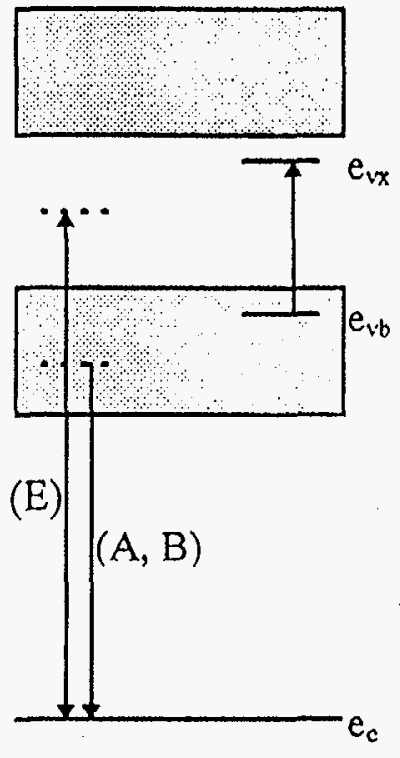

(a)

Inelastic Raman

Scattering

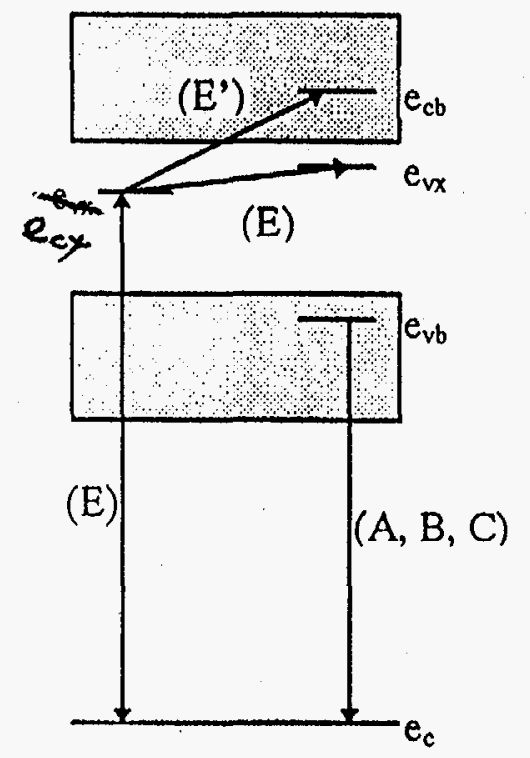

(b)

Spectator

Fluorescence

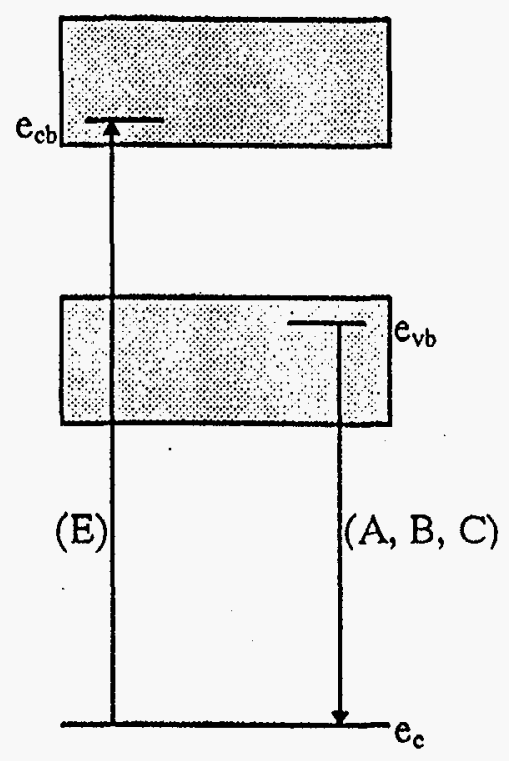

(c)

Normal

Fluorescence

Fig.4 


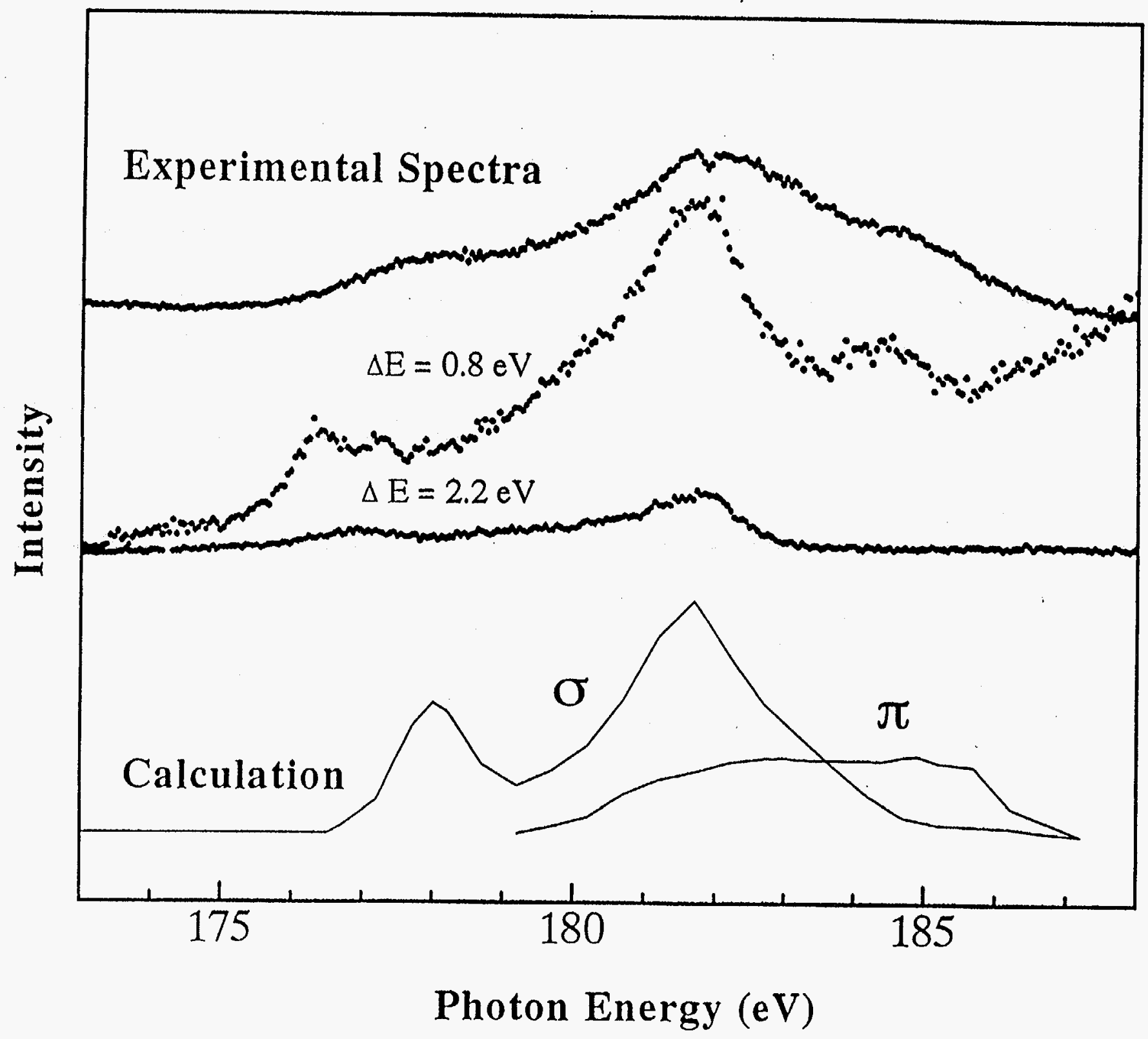

Fig. 5 